\title{
COMPETITIVE OUTCOMES AND ENDOGENOUS COALITION FORMATION IN AN n-PERSON GAME ${ }^{1}$
}

\author{
Ning Sun ${ }^{2}$, Walter Trockel ${ }^{3}$, and Zaifu Yang ${ }^{4}$
}

\begin{abstract}
In this paper we study competitive outcomes and endogenous coalition formation in a cooperative n-person transferable utility (TU) game from the viewpoint of general equilibrium theory. For any given game, we construct a competitive exchange coalition production economy corresponding to the game. First, it is shown that the full core of a TU game is not empty if and only if the completion of the game is balanced. The full core is defined free of any particular coalition structure and the coalitions of the game emerge endogenously from the full core. Second, it is shown that the full core of a completionbalanced general TU game coincides with the set of equilibrium payoff vectors of its corresponding economy and that the coalition structures of the game are endogenously determined by the equilibrium outcomes of the economy. As a consequence, the core of a balanced general TU game coincides with the set of equilibrium payoff vectors of its corresponding economy.
\end{abstract}

Keywords: Cooperative games, endogenous coalition formation, core, full core, equilibrium.

JEL classification: C6, C62, C68, C72, C78.

\footnotetext{
${ }^{1}$ Part of this work was done while the third author was a research fellow of the Alexander von Humboldt Foundation, at the Institute of Mathematical Economics, Bielefeld University, Bielefeld, Germany. He wishes to thank the Institute and the Foundation for the support.

${ }^{2}$ N. Sun, Department of Management Science, Faculty of System Science and Technology, Akita Prefectural University, Honjo, Akita 015-0055, Japan. E-mail: sun@akita-pu.ac.jp

${ }^{3} \mathrm{~W}$. Trockel, Institute of Mathematical Economics, Bielefeld University, 33615 Bielefeld, Germany. E-mail: wtrockel@wiwi.uni-bielefeld.de

${ }^{4}$ Z. Yang, Faculty of Business Administration, Yokohama National University, Yokohama 240-8501, Japan, E-mail: zyang@business.ynu.ac.jp
} 


\section{Introduction}

This paper is concerned with the simultaneous solution of three problems in cooperative game theory, namely what coalitions will form, how these coalitions will allocate their joint payoffs among their members and how these two problems are related to the decentralization of allocation via competitive prices.

According to Zhou (1994) "the first two problems are the two most fundamental questions in cooperative game theory". The third problem may be traced back to the alternative approaches to competitive analysis due to Edgeworth (1881) and Walras (1874) linked by the Core Equivalence Principle.

Modern versions of this principle are theorems due to Debreu and Scarf (1963), Aumann (1964), Anderson (1978) and Wooders (1994). While the first three of these regard (approximate) equivalence of the core and Walrasian allocations in pure exchange economies Wooders' work is closely related to Shapley's and Shubik's $(1969,1975)$ "direct markets" where personal commodities of players allow almost identification of certain markets and games. All this literature, however, based on the standard use of the core and, in Wooders' case, on superadditivity, is not concerned with our first problem of endogenous coalition formation.

It was almost unavoidable that classical game theory largely suppressed the first problem because the usually assumed superadditivity in a context of TU-games made this problem obsolete. Any payoff allocation that appeared desirable for a potential solution could be accomplished by the grand coalition of all players. As superadditivity had made its way also into general NTU-games developed in some articles by Aumann, Peleg and Shapley around 1960 the framework almost determined already the grand coalition as the final active one. This aspect is reflected very clear in the standard definition of the core where the forming of the grand coalition is built in the definition by requiring for any element in the core attainability for the grand coalition. So, whatever payoff allocation in

the core of a game may be realizable by some specific partition of the grand coalition it can as well be realized by the grand coalition itself. Hence there is no need in focussing on the first of the two problems, namely the coalition building. 
The fact that meaningful solutions should allow for the possibility of other coalition structures than the grand coalition has been the cause for a different approach by Thrall and Lucas (1965). However, there the coalition structure is still given exogenously. The need for an endogenously determined coalition structure has been recognized by Aumann and Dreze (1974), who after debating solution concepts with exogenous coalition structure wrote:

"If the reader whishes, he may view the analysis [here] as part of a broader analysis, which would consider simultaneously the process of coalition formation and the bargaining for the payoff..... Our analyis has been concerned with this last topic, and should thus be understood as a contribution to partial equilibrium analysis."

Two decades later Maschler (1992) wrote:

"Consider a group of players who face a game. A basic question would be: What coalitions will form and how will their members share the proceeds? In my opinion, no satisfactory answer has so far been given to this important question. [The current theory] answers a more modest question: How would or should the players share the proceeds, given that a certain coalition structure has formed?"

Zhou (1994) discusses "why there is still not a solution concept that endogenously provides answers to the question of coalition formation as well as that of payoff distribution." After that he formulates three properties such a solution concept should satisfiy: "(1) It is not a priori defined for payoff vectors of a particular coalition structure; (2) it always chooses a nonempty set of some payoff vectors of some coalition structures; and (3) it does not always contain payoff vectors of every coalition structure". He then continues. "Given (1) - (3), such a solution concept will yield endogenously for each game a selected set of coalition structures and payoff vectors consistent with these coalition structures."

But Zhou rather than modifying the use of the core proposes a modification of various concepts of the bargaining set in order to define a solution concept that satisfies his proposed three properties. Yet, in contrast to the core that in large finite economies represents approximately the competitive outcomes of Walrasian equilibria, the Zhou Bargaining Set fails to approximately represent the Walrasian allocations (cf. Anderson, Trockel and Zhou (1997)). It is therefore not suitable for approaching our third problem of relating cooper- 
ative behavior as represented by the solution concept with competitive allocations of the economy.

Yet, there are already some examples for such solutions in the literature. Neuefeind (1974) and Böhm (1974) in different contexts define a core without requiring that the set of all possible allocations coincides with the set of those allocations that are feasible for the grand coalition. While Böhm does not specify that set of generally possible allocations Neuefeind explicitly defines it as the union of all allocations feasible for any coalition structure. Such a core is consistent with the three properties formulated by Zhou.

The core as a solution, despite its non-existence for certain classes of games, in market games, when it exists, is often quite large. But market games allow it to look at the competitive payoffs generated in the underlying economy or market that build a subset of the core.

Shapley and Shubik (1975, section 3) write "The so called "competitive solution" is not a game theory concept, but is based on the notion of prices ... "clearing the market" to everyone's satisfaction". But for market games such competitive payoffs build appealing points of reference that suggest themselves for comparison with game theoretic solution concepts like the (inner; strong) core.

As Shapley and Shubik (1975) show for TU market games and Qin (1993) for NTU market games several markets usually are represented by the same game but may differ considerably in their sets of competitive allocations.

Two extreme cases are the coincidence of the set of competitive payoffs with the core (resp. the inner core for NTU games) or, coincidence only with one prespecified payoff allocation in the core (resp. inner core). This fact has been interpreted by Shapley and Shubik (1975) as a loss of potentially valuable information on the economic system by passing to a representing game.

These considerations regard game theoretical modelling as a methodological tool chest for economics, in particular, general equilibrium analysis. That is also largely the case for the approach of Wooders (1994). But there is also the converse direction that aims to analyze cooperative games via tools from economic theory, as for instance equilibrium price systems. Shapley and Shubik (1975) hint to that direction when they introduce their 
concept of a "direct market" in their section 4.1. In fact they start this section with the following passage:

"Thus far we used markets to generate games. We now go the reverse route, associating with any game (not necessarily a market game) a certain "market of coalitions"." This comes close to looking at games as markets and to defining competitive allocations in games. The same view is expressed by Wooders (1994, p. 1143) who writes: "The research noted above raises the possibility not only that large games and economies ... behave like competitive markets but also that these games and economies are competitive markets or close to them."

In fact there is an instant where prices appear explicitly in games, namely as defining specific TU-games as $\lambda$-transfers of NTU-games (cf. Shapley (1969), Qin (1993)). And Shubik (1984, p. 192) hints to the similarity of those $\lambda$ 's to competitive price systems and asks for a model in which this relation is formally established. This problem of Shubik had been solved in Trockel (1996) where the Nash solution that coincides with the $\lambda$-transfer value on bargaining games has been established as a competitive payoff allocation where the supporting vector $\lambda$ is a corresponding equilibrium price vector in the game interpreted as an Arrow-Debreu economy.

It is our goal in the present paper, deviating from most of the literature on market games, to represent any given TU-game by a coalition production economy in the tradition of McKenzie (1981), Böhm (1974), and Sondermann (1974) and to relate the resulting equilibrium payoffs to the core. But in order to enable the specific equilibria to endogenously determine the formation of coalitions we modify the core concept. We define the full core of a TU-game $v$ as the core of its completion $\hat{v}$ which coincides with $v$ on all coalitions except the grand one but associates with the grand coalition the maximal sum of coalitions' worths that a partition could provide. This corresponds exactly to the core in Neuefeind (1974) but here in a TU-context.

Rather than interpreting our full core as a new solution concept one should think of it as enlarging the set of principally possible payoff vectors and making it the new set of payoff allocations feasible for the grand coalition.

The methodological contributions of our paper as compared with the existing literature 
are the following:

.) We extend the usual market representations of games that are pure trade models or models where each trader has his own technology by a coalition production economy where each coalition owns a production possibility set.

-) We consider for arbitrary games $v$ their completions and thereby create the framework in that, despite the use of the core concept, we allow for endogenous determination of coalitions.

Then applying the Bondareva-Shapley Theorem on our new class of complete games we prove that the full core of any game coincides with the set of equilibrium payoff vectors of the induced coalition production economy provided the completion of the game is balanced. Moreover, any Walrasian equilibrium determines endogenously an associated coalition structure for the game.

Formally our theorem can be seen as an extension of Shapley's and Shubik's (1975) first theorem from direct market to coalition production economies. It is this richer economic framework that enables us to endogenously determine equilibrium coalition structures.

The rest of this paper is organized as follows. In Section 2, the full core is defined and a necessary and sufficient condition for its existence is formulated. The main results of this paper are established in Section 3.

\section{Coalition formation and full core}

Some general notation is needed in the sequel. For a finite set $K$, the set $\mathbb{R}^{K}$ denotes the $|K|$-dimensional Euclidean space where the coordinates are indexed by the elements in $K$. Given $x, y \in \mathbb{R}^{K}, x \cdot y$ denotes the inner product of $x$ and $y$. Let $N=\{1,2, \cdots, n\}$ and let $e(i)$ be the ith unit vector of $\mathbb{R}^{N}$. For any subset $S$ of $N$, let $e(S)=\sum_{i \in S} e(i)$. For any subset $S$ of $N$ and any element $x$ in $\mathbb{R}^{N}$, let $x(S)$ be equal to $\sum_{i \in S} e(i) x_{i}$, where $x=\left(x_{1}, x_{2}, \cdots, x_{n}\right)$.

Let $N=\{1,2, \cdots, n\}$ be the set of players and let $2^{N}$ be the collection of all nonempty coalitions, $S \subset N$. An n-person transferable utility (TU) game is defined by $G=(N, V)$, 
where $V$ is a function from $2^{N}$ to subsets of $\mathbb{R}^{N}$. For each coalition $S, V(S)$ is a set of payoff vectors which players in the coalition $S$ can achieve by acting together. It is assumed that for every coalition $S$, there exists a nonnegative real number $v(S)$ such that $V(S)=\left\{x \in \mathbb{R}^{N} \mid \sum_{i \in S} x_{i} \leq v(S)\right\}$. In the following we use $(N, V)$ and $(N, v)$ interchangeably.

A very intuitive and fundamental solution concept for cooperative games is the core. The core of an $n$-person TU game $(N, V)$ is defined to be the set of elements $x$ in $V(N)$ so that there exists no coalition $S$ such that there is some $y \in V(S)$ satisfying $y_{i} \geq x_{i}$ for every $i \in S$ and $y_{j}>x_{j}$ for some $j \in S$.

Recall that a family $\mathcal{B}$ of coalitions in a game is called a balanced family if there exist nonnegative numbers $\delta_{S}$, for each coalition $S$ in $\mathcal{B}$, such that

$$
\sum_{i \in S \in \mathcal{B}} \delta_{S}=1, \text { for every i } \in N
$$

A game $(N, V)$ is called a balanced game if for every balanced family $\mathcal{B}$ of coalitions it holds

$$
\sum_{S \in \mathcal{B}} \delta_{S} v(S) \leq v(N)
$$

A balanced game is said to be totally balanced if all of its subgames, obtained by restricting $v$ to the subsets of $S, S \subset N$, are also balanced. It is well known that a balanced NTU game has a non-empty core (cf. Scarf (1967)) and that for TU games also the converse is true (cf. Shapley (1967)). Shapley and Shubik show that their market games are exactly the totally balanced TU games (cf. Shapley and Shubik(1969)) and analyze for these games the relation between the core and competitive payoff vectors (cf. Shapley and Shubik(1975)).

Interesting as it is, the core requires that the grand coalition $N$ must be formed if a game is played. In this paper one of our objectives is to provide answers endogenously to the question of coalition formation. For this purpose, we have to consider the situtation where for one reason or another several coalitions may emerge if a game is played.

A family $\pi=\left\{S_{1}, S_{2}, \cdots, S_{t}\right\}$ of $t$ coalitions is called a partition of the set $N$ if $S_{i} \cap S_{j}=\emptyset$ for $i \neq j, \cup_{j=1}^{t} S_{j}=N$, and $S_{j} \neq \emptyset$ for $j=1, \cdots, t$. When $t=1$, then we have the grand coalition $N$; when $t=n$, each individual player is acting independently. Let $\mathcal{P}$ denote the 
family of all partitions $\pi$. Given a game $G=(N, V)$, we define an induced game $(N, \hat{V})$ or $(N, \hat{v})$ where

$$
\hat{V}(N)=\cup_{\pi \in \mathcal{P}} \cap_{S \in \pi} V(S), \hat{v}(N)=\max _{\pi \in \mathcal{P}} \sum_{S \in \pi} v(S),
$$

and

$$
\hat{V}(S)=V(S), \quad \hat{v}(S)=v(S), \forall S \subset N \text { with } S \neq N
$$

and call the induced game the completion of the game $(N, V)$. The core of the complete game $(N, \hat{V})$ will be called the full core of the original game $(N, V)$. Note that the full core is defined free of any particular coalition structure. Therefore, if the full core is nonempty, then the coalitions of the game will emerge endogenously when the game is played.

Now we will briefly review several closely related works. Aumann and Dreze (1974) study various solution concepts with exogenous coalition structures. Mas-Colell (1989) and Zhou (1994) have proposed two different bargaining solutions independent of any coalition structure and proved their existence for general games. However, it appears that their bargaining solutions are so weak that they cannot be used to achieve our major goal, namely, the competitive outcomes (cf. Anderson, Trockel and Zhou (1997)). Nevertheless, keep in mind that the emphasis of Mas-Colell's and Zhou's papers is quite different from ours.

A game $(N, V)$ is called a c-balanced game (meaning completion-balanced) if its completion $(N, \hat{V})$ is balanced. As an immediate consequence of the well-known BondarevaShapley core existence theorem, we have the following observation that is crucial for the existence of a full core in every c-balanced game:

A TU game has a nonempty full core if and only if it is c-balanced.

\section{The stylized economy and competitive outcomes}

Given a TU game $G=(N, V)$, we construct a coalition production economy corresponding to the game:

$$
E(G)=\left\{\left(X^{i}, u^{i}, \omega^{i}, i \in N\right),\left(Y^{S}, S \in 2^{N}\right)\right\}
$$


where $X^{i}=\mathbb{R}_{+}^{N} \times \mathbb{R}_{+}^{N}$ is the consumption set for agent $i \in N$, here for $(x, y) \in X^{i}, x$ is viewed as input goods and $y$ as output or final goods; agent $i^{\prime} s$ utility function $u^{i}$ : $\mathbb{R}^{N} \times \mathbb{R}^{N} \rightarrow \mathbb{R}$ is specified by $u^{i}(x, y)=y_{i}$, namely, agent $i$ is only interested in output good $i$; agent $i$ is initially endowed with the bundle $\omega^{i}=(e(i), 0)$; to each coalition $S$ there is associated the production set

$$
Y^{S}=\left\{(x, y) \in \mathbb{R}^{N} \times \mathbb{R}^{N} \mid x \in-\mathbb{R}_{+}^{N}, x_{i}=y_{i}=0 \text { if } i \notin S, \sum_{h \in S} y_{h} \leq \min _{h \in S}\left|x_{h}\right| \hat{v}(S)\right\} .
$$

Thus, there are $n$ consumers, who are the $n$ players, $n$ input goods, $n$ output goods, and $2^{n}-1$ production sets in the economy. This coalition production economy is a simple but slightly modified version of McKenzie models (cf. McKenzie $(1959,1981)$ ).

See also Böhm (1974), Hildenbrand (1974), and Sondermann (1974)), for related models. Note that in the economy each coalition $S$ can be seen as a firm that is controlled by its members. By definition, here every production set exhibits constant returns to scale. Furthermore, it should be noted that in the economy, the ownership structure of each firm is not exogenously given but will rather be endogenously determined by the equilibrium outcomes.

Given a price vector $(q, p) \in \mathbb{R}_{+}^{N} \times \mathbb{R}_{+}^{N}$, each consumer $i$ will choose an optimal consumption plan to maximize her utility under her budget constraint and each firm $S$ will select an optimal production plan to maximize its profit. More precisely, consumer $i$ solves the problem

$$
\begin{array}{cc}
\max & u^{i}(x, y) \\
\text { s.t. } & (q, p) \cdot(x, y) \leq(q, p) \cdot \omega^{i} \\
& (x, y) \in \mathbb{R}_{+}^{N} \times \mathbb{R}_{+}^{N} .
\end{array}
$$

Due to the simple form of $u^{i}$ and $\omega^{i}$, the above problem is reduced to

$$
\begin{array}{cc}
\max & y_{i} \\
\text { s.t. } & p_{i} y_{i} \leq q_{i}
\end{array}
$$

Firm $S$ solves the problem

$$
\begin{array}{cc}
\max & (q, p) \cdot(x, y) \\
\text { s.t. } & (x, y) \in Y^{S} .
\end{array}
$$


An equilibrium of the economy is a state in which every consumer obtains an optimal consumption bundle under her budget constraint and every firm achieves maximal profit and the market is clear. Formally, the equilibrium concept is defined as follows.

Definition 3.1 A list of vectors

$$
\left\{(q, p),\left[\left(0, z^{i}\right), i \in N\right],\left[\left(x^{S}, y^{S}\right) \in Y^{S}, S \in 2^{N}\right]\right\}
$$

is a Walrasian equilibrium if the following conditions are satisfied:

(1) $(q, p) \in \mathbb{R}_{+}^{N} \times \mathbb{R}_{+}^{N}$;

(2) for every $S \in 2^{N}$,

$$
q \cdot x^{S}+p \cdot y^{S}=\max _{(x, y) \in Y^{S}}(q \cdot x+p \cdot y)=0
$$

(3) $u^{i}\left(0, z^{i}\right)=z_{i}^{i}=\max \left\{z_{i}^{\prime} \mid p_{i} z_{i}^{\prime} \leq q_{i}\right\}$

(4) $\sum_{i \in N}\left(0, z^{i}\right)=\sum_{S \in 2^{N}}\left(x^{S}, y^{S}\right)+\sum_{i \in N} \omega^{i}$;

(5) for every $S \in 2^{N}, x^{S}$ is an element in $\{-1,0\}^{N}$.

Note that in equilibrium, no production activity makes profit due to constant returns to scale. The parameter $\left|x_{i}^{S}\right|$ for every $i \in S$ can be interpreted as the portion of consumer $i^{\prime} s$ willingness or investment to contribute to the firm $S$. In equilibrium, $\sum_{i \in N} z^{i}$ is called the equilibrium payoff vector, $(q, p)$ the equilibrium price vector, and $\left(x^{S}, S \in 2^{N}\right)$ the equilibrium coalition structure. Clearly, an equilibrium coalition structure $\left(x^{S}, S \in 2^{N}\right)$ defines a partition $\pi=\left\{O_{1}, \cdots, O_{t}\right\}$ of the grand coalition $N$ for some positive integer $t$. Namely, $S \in \pi$ if and only if $x_{i}^{S}=-1$ for every $i \in S \neq \emptyset$, and for every $T \supset S, T \neq S$, there is some $j \in T$ such that $x_{j}^{T}=0$.

We are now ready to present and prove the main result of this paper.

Theorem 3.2 The full core of a c-balanced TU game coincides with the set of equilibrium payoff vectors of the corresponding coalition production economy. Furthermore, the final coalitions of the game are endogenously determined by the equilibrium coalition structures of the economy. 
Proof: Let $z$ be an element of the full core of the TU game $G=(N, V)$. We will prove that $z$ is an equilibrium payoff vector of the corresponding economy. Since $z$ is an element of the full core, there exists a partition $\pi \in \mathcal{P}$ such that $z \in \cap_{S \in \pi} V(S)$. Let $y^{S}=z(S)$ and $x^{S}=-e(S)$ for every $S \in \pi$ and $y^{S}=0$ and $x^{S}=0$ otherwise. Let $p=e(N), q=z$, $\omega^{i}=(e(i), 0)$ and $z^{i}=z_{i} e(i)$ for every $i \in N$. We will show that

$$
\left\{(q, p),\left[\left(0, z^{i}\right), i \in N\right],\left[\left(x^{S}, y^{S}\right) \in Y^{S}, S \in 2^{N}\right]\right\}
$$

constitutes an equilibrium. Clearly, conditions (1), (3), (4), (5) of Definition 3.1 are satisfied. It remains to check condition (2). For every $S \in 2^{N}$, it is obvious that $p \cdot y^{S}+q \cdot x^{S}=0$. We still have to show that

$$
\max _{(x, y) \in Y^{S}}(q \cdot x+p \cdot y)=0 .
$$

Let $(x, y) \in Y^{S}$. We can write $(x, y)=\left(-\lambda_{S} e(S), y\right)+\left(\lambda_{S} e(S)+x, 0\right)$, where $\lambda_{S}=$ $\min _{i \in S}\left|x_{i}\right|$. It follows that

$$
\begin{aligned}
q \cdot x+p \cdot y & =e(N) \cdot y+z \cdot x \\
& \leq e(N) \cdot y-z \cdot \lambda_{S} e(S) \\
& =\sum_{i \in S} y_{i}-\lambda_{S} \sum_{i \in S} z_{i} \\
& \leq \lambda_{S} \hat{v}(S)-\lambda_{S} \sum_{i \in S} z_{i} \\
& =\lambda_{S}\left(-\sum_{i \in S} z_{i}+\hat{v}(S)\right) \\
& \leq 0 .
\end{aligned}
$$

Note that to derive the above inequalities we have used the facts that $\lambda_{S} e(S)+x \leq 0$, $y_{i}=0$ for every $i \notin S, \sum_{i \in S} y_{i} \leq \lambda_{S} \hat{v}(S)$, and $\sum_{i \in S} z_{i} \geq \hat{v}(S)$.

Now suppose that

$$
\left\{(q, p),\left[\left(0, z^{i}\right), i \in N\right],\left[\left(x^{S}, y^{S}\right) \in Y^{S}, S \in 2^{N}\right]\right\}
$$

is an equilibrium. Let $z=\sum_{i \in N} z^{i}$. We will show that $z$ is an element of the full core of the underlying game. First, note that because the game is a transferable utility game, every component of the equilibrium price vector $p$ must be equal and positive. Thus, without loss of generality we can assume that $p=e(N)$. Thus, $q=z$. Note that $\sum_{S \in 2^{N}} x^{S}+e(N)=0$ and $\sum_{S \in 2^{N}} y^{S}=z$. Let $\lambda_{S}=\min _{i \in S}\left|x_{i}^{S}\right|$ for every $S \in 2^{N}$. We can write that $\left(x^{S}, y^{S}\right)=$ 
$\left(-\lambda_{S} e(S), y^{S}\right)+\left(x^{S}+\lambda_{S} e(S), 0\right)$. Clearly, by definition $\left(-\lambda_{S} e(S), y^{S}\right) \in Y^{S}$. Therefore, $\sum_{i \in S} y_{i}^{S} \leq \lambda_{S} \hat{v}(S)$. Note that $\sum_{S \in 2^{N}} \lambda_{S} e(S) \leq e(N), y_{i}^{S}=0$ for $i \notin S$, and that the game is complete balanced. We have

$$
\begin{aligned}
\sum_{i \in N} z_{i} & =\sum_{i \in N} \sum_{S \in 2^{N}} y_{i}^{S} \\
& =\sum_{S \in 2^{N}} \sum_{i \in S} y_{i}^{S} \\
& \leq \sum_{S \in 2^{N}} \lambda_{S} \hat{v}(S) \\
& \leq \hat{v}(N) .
\end{aligned}
$$

On the other hand, for every $(-e(S), y) \in Y^{S}$, it follows from condition (2) of Definition 3.1 that

$$
\begin{aligned}
0 & \geq p \cdot y+q \cdot(-e(S)) \\
& =e(N) \cdot y+z \cdot(-e(S)) \\
& =\sum_{i \in S} y_{i}-\sum_{i \in S} z_{i} .
\end{aligned}
$$

This implies that

$$
\sum_{i \in S} z_{i} \geq \sum_{i \in S} y_{i}
$$

for every $(-e(S), y) \in Y^{S}$. Therefore, we have

$$
\sum_{i \in S} z_{i} \geq \hat{v}(S)
$$

for every $S \in 2^{N}$. In conclusion, $z$ is an element of the full core.

As a consequence, there exists some partition $\pi$ such that $z \in \cap_{S \in \pi} V(S)$. This partition $\pi$ is exactly the one that emerges from the equilibrium outcomes. Namely, $\pi$ corresponds to the equilibrium coalition structure, $\left(x^{S}, S \in 2^{N}\right)$.

As a corollary, we have:

Theorem 3.3 The core of a balanced TU game coincides with the set of equilibrium payoff vectors of the corresponding coalition production economy.

\section{References}

[1] R.J. Anderson, W. Trockel and L. Zhou: "Nonconvergence of Mas-Collel's and Zhou's Bargaining Sets," Econometrica, 65 (1997), 1227-1239. 
[2] R.J. Aumann and J.H. Dreze: "Cooperative games with coalition structures," International Journal of Game Theory, 3 (1974), 217-237.

[3] R.J. Aumann and B. Peleg: "Von Neumann-Morgenstern solutions to cooperative games without side payments," Bulletin of the American Mathematical Society, 66 (1960), 173-179.

[4] R.J. Aumann: "A Survey of Cooperative Games without Side Payments," in: Essays in Mathematical Economics in Honour of Oskar Morgenstern (M. Shubik, Ed.), Princeton University Press, Princeton, New Jersey, pp. 3-27, 1967.

[5] R.J. Aumann: "Markets with a Continuum of Traders," Econometrica, 32 (1964), 39-50.

[6] V. Böhm: "The core of an economy with production," Review of Economic Studies, 41 (1974), 429-436.

[7] G. Debreu and H. Scarf: "A limit theorem on the core of an economy," International Economic Review, 4 (1963), 235-246.

[8] F.Y. Edgeworth: Mathematical Psychics, Paul Kegan, London, 1881.

[9] W. Hildenbrand: Core and Equilibria of a Large Economy, Princeton University Press, Princeton, New Jersey, 1974.

[10] M. Maschler: "The Bargaining Set, Kernel and Nucleolus: A Survey," in: Handbook of Game Theory, Vol. 1, (R. Aumann and S.Hart, Eds.), Elsevier, Amsterdam, pp. 591-667, 1992.

[11] A. Mas-Colell: "An equivalence theorem for a bargaining set," Journal of Mathematical Economics, 18 (1989), 129-139.

[12] L.W. McKenzie: "On the existence of general equilibrium for a competitive market," Econometrica, 27 (1959), 54-71.

[13] L.W. McKenzie: "The classical theorem on the existence of competitive equilibrium," Econometrica, 49 (1981), 819-841. 
[14] W. Neuefeind: "A stochastic bargaining process for n-person games," Journal of Mathematical Economics, 1 (1974), 175-191.

[15] C. Qin: "A conjecture of Shapley and Shubik on competitive outcomes in the cores of NTU market games," International Journal of Game Theory, 22 (1993), 335-344.

[16] H. Scarf: "The core of an n person game," Econometrica, 35 (1967), 50-69.

[17] L.S. Shapley: "On balanced sets and cores," Naval Research Logistics Quarterly, 14 (1967), 453-460.

[18] L.S. Shapley: "Utility Comparison and the Theory of Games," in: La Decision: aggrgation et dynamique des ordres de preference, Paris, pp. 251-263, 1969.

[19] L.S. Shapley: "Notes on the inner core," Lecture Notes, University of California at Los Angeles, Los Angeles, 1984.

[20] L.S. Shapley and M. Shubik: "On market games," Journal of Economic Theory, 1 (1969), 9-25.

[21] L.S. Shapley and M. Shubik: "Competitive outcomes in the cores of market games," International Journal of Game Theory, 4 (1975), 229-237.

[22] M. Shubik: Game Theory in Social Sciences: Concepts and Solutions, MIT Press, Cambridge, 1984.

[23] D. Sondermann: "Economics of scale and equilibria in coalition production economies," Journal of Economic Theory, 8 (1974), 259-291.

[24] R.M. Thrall and W.F. Lucas: " $N$-person games in partition function form," Naval Research Logistics Quarterly, 10 (1963), 281-298.

[25] W. Trockel: "A Walrasian approach to bargaining games," Economics Letters, 51 (1996), 295-301.

[26] L. Walras: Eléments d'Economie Politique Pure, L. Corbaz, Lausanne, 1874. 
[27] M. Wooders: "Equivalence of games and markets," Econometrica, 62 (1994), 11411160.

[28] L. Zhou: "A new bargaining set of an $N$-person game and endogenous coalition formation," Games and Economic Behavior, 6 (1994), 231-246. 